\title{
II. \\ Über die Stellung der Tauner nach den Rechtsquellen des Kantons Zürich.
}

\author{
Von \\ Herrn Dr. jur. Ed. Eichholzer \\ in Bern.
}

I. Die Tauner spielen in der Rechts- und Wirtschaftsgeschichte der ebenen Landesteile der Schweiz eine nicht unbedeutende Rolle. Es hält schwer, die Stellung des Tauners begrifflich zu umschreiben. Es geht nicht an, den Tauner, wie ihn die Quellen uns schildern, ohne weiteres dem modernen Tagelöhner gleichzusetzen. ${ }^{1}$ ) Eher läßt er sich mit den Häuslern, Büdnern, Kossäten der deutschen Wirtschaftsgeschichte vergleichen, wobei allerdings die besonderen schweizerischen Verhältnisse zu berücksichtigen sind.

Die Bezeichnung ,Tauner" wird heute in der in Betracht kommenden Literatur vorwiegend gebraucht ${ }^{2}$ ), doch ist dies nicht durchweg der Ausdruck der Rechtsquellen, auf alle Fälle nicht derjenigen aus dem Kanton Zürich. Die älteste uns bekannte zürcherische Rechtsquelle, welche von den Taunern handelt, ein Erkenntnis von Bürgermeister und Rat der Stadt Zürich vermutlich aus den Jahren 1485 bis 1489 über die Benützung der Allmenden ${ }^{3}$ ), nennt dieselben ,tagwner". Am häufigsten jedoch sind im Gebiete des Kantons Zürich die Bezeichnungen Tagner und Tagnauer (,tagnower", ,tagnouwer", „,tagnöwer"). Der Aus-

1) Als Tagelöhner wird der Tauner im deutschen Wörterbuch von J. und W. Grimm bezeichnet (Bd. XI S. 88).

2) Dies nicht nur in der Fachliteratur, sondern auch in der schönen Literatur. Mit Bezug auf letzteres vgl. die Nachweise im Grimmschen Wörterbuch a. a. O. aus den Werken H. Pestalozzis und Jeremias Gotthelfs.

3) Abgedruckt in der Zeitschrift für noch ungedruckte Schweiz. Rechtsquellen, herausg. von Schauberg, Bd. I S. I36f. 
druck Tauner scheint hauptsächlich in den westlichen Teilen der deutschen Schweiz gebraucht worden zu sein. ${ }^{1}$ ) Die ursprüngliche Wortform ist ,Tagwener", ,Tagwaner". Die Tauner hängen denn auch sachlich mit dem in mehrfacher Bedeutung vorkommenden Worte Tagwen zusammen.

Tagwen bedeutet in erster Linie eine durch den Zeitraum eines Tages begrenzte Arbeit, geleistet im Interesse irgendeiner Obrigkeit als Frondienst, oder im gemeinen Interesse oder endlich um des Verdienstes willen. ${ }^{2}$ ) Im letzteren Falle ist Tagwen gleichbedeutend mit Arbeit im Tagelohn. Das entsprechende Zeitwort lautet ,tagmen“3), „taune“.4) Nach den Quellen wird zwar der Ausdruck Tagwen sowohl für Handdienste als auch für Spanndienste gebraucht.5) Allein, gleich wie der Ausdruck Tagelöhner bezeichnend geworden ist für einen Landarbeiter, der lediglich seine eigene Körperkraft in den Dienst der Arbeit stellt, so ist es auch mit dem Ausdruck Tagwen ergangen. „Tagwen“ wurde in erster Linie die Bezeichnung für durch der Hände Arbeit geleistete Dienste. Es ist nun begreiflich, daß vor allem der Kleinbauer und Nichtviehbesitzer die schuldigen Dienste nur durch. Handarbeit leisten konnte. Es erscheint daher als naheliegend, daß auf diese Weise die ganze in Frage stehende soziale Schicht den Namen Tagwener erhielt. So wird denn auch heute die Tatsache, daß der Tauner im Gegensatz zu den Besitzern von Zugvieh dem Herrn oder dem Gemeinwesen nur Handdienste leisten konnte, als ein maßgebendes Unterscheidungsmerkmal zwischen dem Stand der Tauner und demjenigen der

1) Über dem Tauner entsprechende Ausdrücke aus den Rechtsquellen der französischen Schweiz vgl. H. Rennefahrt, Die Allmend im Berner Jura (Heft 74 der Gierkeschen Untersuchungen) S. 37 Anm. 1.

2) Vgl. über den Ursprung des Wortes Tagwen das Grimmsche Wörterbuch Bd. XI S. 87.

3) Siehe Stalder, Schweiz. Idiotikon I S. 258.

4) Siehe Hunziker, Aargauer Wörterbuch S. 47.

5) Z. B. Offnung von Flaach (Grimm, Weistümer Bd. I S. 94): „Item alle züg, so zu Flaach unnd Follckenn uß gand, sol yeder tzug eynem vogtherren deß yars ein tauwen thun mit dem zug, unnd darnach alle die, die yer eygens brott essen zu Flach unnd Folcken sol ein yeder dem vogterren zu Flach alle iar ein tauwen tun mit der Hand." 
Die Stellung d. Tauner n. d. Rechtsquellen d. Kantons Zürich. 117

„,Bauern“" aufgefaßt. ${ }^{1}$ ) So geschieht dies in den grundlegenden Abhandlungen von Renaud über die Gemeindenutzungen ${ }^{2}$ ) und von $F$. v. Wy $B$ über die schweizerischen Landgemeinden in ihrer historischen Entwicklung. ${ }^{3}$ )

Es ist in diesem Zusammenhang noch zu erwähnen; daß das Wort Tagwen, wie wir es oben kennengelernt haben, nicht nur den Taunern den Namen gab, sondern auch den heute noch ,Tagwen" genannten öffentlich-rechtlichen Korporationen im Kanton Glarus. Auch hier bildete der Frondienst den Ausgangspunkt. Die glarnerischen Tagwen sind, wie Stüssi in seiner Geschichte des glarnerischen Land- und Tagwenrechts ausführt ${ }^{4}$ ), aus Frondienstgemeinschaften entstanden.

II. Die Tauner waren mit ihrer schwächeren Arbeitsund Steuerkraft für denjenigen, dem die Frondienste oder die Abgaben entrichtet werden mußten, von geringerer wirtschaftlicher Bedeutung als der eigentliche Bauernstand. Es liegt auf der Hand, daß diese verschiedene Bewertung der Tauner und der „Bauern“ - womit wir in Übereinstimmung mit den Quellen die zu den Taunern im Gegensatz stehenden Landwirte bezeichnen wollen - da praktisch werden mußte; wo die Landbewohner, mehr oder weniger als Äquivalent für ihre Dienste, etwas bezogen, nutzungsberechtigt waren. ${ }^{5}$ ) Es kam hier vor allem die Nutzung am All-

1) Vgl. hierzu folgende Stellen aus den Quellen: Offinung von Wülflingen, 1484 (Grimm, W. I S. 138): ,Item einer der da seßhaft ist, und ist er ein tauwner, so sol er dem vogt des jabrs ein tauwen thun, und im damit han gedienet, hatt er aber einen zug, so sol er im einen tag ehren, und hat im auch damit gedienet." - Holzordnung aus Affoltern a. A. (Rechtsquellen des Kantons Zürich, 1. Teil, Offnungen und Hofrechte, herausg. von R. Hoppeler, Bd. I S. 80 § 9): ,, Sovil dann bruggen, wägen und der gmeind zünung als gmeine werch antrifft, da söllind die puren und die, so roß und fech habind, jeder zyt zu demselben allem alle nothurfft, holtz, stein und ander zu hin ze füren schuldig sin, und dann die tagner all gemeinlich mit iren lyben ire tagwan und ir bests thun."

2) Zeitschrift für deutsches Recht Bd. IX S. 36.

3) Neu herausgegeben in den Athandlungen zur Geschichte des schweiz. öffentl. Rechts, 1892, S. 104.

4) Zürcher jur. Diss., 1912, S. 5-7.

$\left.{ }^{5}\right)$ Vgl. Hotz, Rechtsverhältnisse an der Almende Horger-Egg, 1866, S. 14. 
mendland in Frage. Dis Stellung der Tauner im Hinblick auf die Allmende ist das wichtigste Problem, welches die Erscheinung der Tauner überhaupt gezeitigt hat.

Allgemein ist zu sagen, daß die Nutzung der Tauner am Allmendland zwar gewöhnlich beschränkt, dagegen soweit es sich nicht um Allmenden handelte, die zum vornherein nur bestimmten Gütern dienten - keineswegs etwa prinzipiell ausgeschlossen war. Die Einteilung der Landbewohner in Baucrn und Tauner fällt nicht mit derjenigen in Genossen und Ungenossen zusammen. Der Nutzungsanspruch des Tauners steht lediglich demjenigen des bevorzugten Bauern nach. In seinen Institutionen des Deutschen Privatrechts I S. 291 vergleicht Heusler diesen verschiedenartigen Umfang der Nutzungen der Genossen treffend mit den Prioritätsaktien und nachgehenden Aktien des modernen Rechtes. Zu einer verschiedenen Bemessung der Nutzungsanteile ist nun allerdings zunächst noch nicht überall Veranlassung vorhanden gewesen, namentlich dort nicht, wo die Allmende noch groß genug war, um allen Bedürfnissen zu genügen. So heißt es beispielsweise in der Offnung vonBassersdorf: „Item es sol ouch die almend alz fry sin, das ein jeglicher, so inwendig etters gesessen ist, daruff sol und mag triben nach dem als er statt hat, einer alz der ander."1) Ja, es kam sogar vor, da $B$ man, wie Graf in seiner Abhandlung über die Aufteilung der Allmend in der Gemeinde Schötz (Luzern) ausführt ${ }^{2}$ ), die Ansiedelung der Tauner, welche man als Arbeitskraft bei der Bewirtschaftung des Sonderbesitzes der Bauern brauchte, direkt dadurch begünstigte, daß man ihnen neue Einschläge auf der Allmend gewährte und ihnen crlaubte, sich auf Allmendboden häuslich niederzulassen.

Mit Bezug auf die zu leistenden Spanndienste wurde nicht nur zwischen Bauern und Taunern unterschieden, man kannte auch noch Halbbauern. Der Bauer mußte mindestens einen Zug haben. Als Zug galten nach einem Ratserkenntnis betreffend den Weidgang der Bauern und Tauner in Affoltern a. Albis vom Jahre 16744 Pferde oder

1) Rechtsquellen des Kantons Zürich, 1. Teil, I S. 391, 47.

2) Bern 1890, S. 30 . 
Die Stellung d. Tauner n. d. Rechtsquellen d. Kantons Zürich. 119

4 Stück Rindvieh oder 4 Stück beider Tierarten. ${ }^{1}$ ) Es kam vor, daß die Bauern direkt ,Züger“ genannt wurden, so in dem Spruch betreffend Steuer und Weidgang der Bauern und Tauner in Rieden vom Jahre 1620. ${ }^{2}$ ) Die Antipoden dieser Züger waren hier ,die, so keine züg habendt und tagnoüwer genempt werdent". Bemerkenswert ist die Abstufung der Allmendnutzung nach der Anzahl des Zugviehs in dem schon erwähnten Ratserkenntnis betreffend den Weidgang der Bauern und Tauner in Affoltern a. Albis vom 18. Februar 1674, weil daraus hervorgeht, daß selbst der Besitz bestimmter Rechtsansprüche auf die Allmendnutzung, sogenannter Gerechtigkeiten, nur eine quantitativ sehr beschränkte Nutzungsbefugnis in sich schließt, wenn sich der Berechtigte nebenbei nicht noch über den Besitz von Zugvieh ausweisen kann. Es heißt da $^{3}$ ): ,Erstlich daß zu Affholtern ein jeder pur, so ein gantzen zug, benanntlich vier haubt roß oder rindervych oder beider gattung, vermag und ein gantze grechtigkeit besitzt, befüegt syn und gwalt haben, zwo küey uff die allment und drü haubt zug vych, under denen höchstens zwey roß, uff die esch zu lassen. - Demnach, wellicher einen halben zug, als zwei gute zughaubt vych, und darnebent ein gantze grechtigkeit hat, zwo küey uff die allment und ein roß oder rind uff die esch lassen mögen, in dem ußtrucklichen verstand, daß die, so solliche gantze und halbe züg habend und vermelter maßen des weidgangs genoß syn wollend, dieselben $u ß$ ihrem eignen futer, so ihnen im Affholtrer zwing wachst, sollind wintern können. Drittens. Wellicher ein gantze grechtigkeit besitzt und zwo küey winteren kan, soll derselbe beid uff die allment lassen, und so er noch darzu ein roß vermöchte, er dasselbe uff die esch schlachen, hingegen dann nur ein ku uff die allment lassen mögen. Vierdtens. Wellicher ein gantze grechtigkeit hat und mehr nit als ein

1) Rechtsquellen des Kantons Zürich, 1. Teil, I S. 87, 1. Dabei wurden allerdings die Kühe nicht als Zugtiere bewertet, wie aus den Quellen verschiedentlich hervorgeht. Unter Rindvieh sind offenbar nur Ochsen zu verstehen.

2) Ebenda, 1. Teil, II S. $405 \mathrm{ff}$.

3) Ebenda 1, Teil, I S. 87/88. 
ku vermag, der soll dieselbe uff die allment lassen, syn grechtigkeit aber zu einem rind uff die esch verliehen oder durch ein entlehnendes vych nutzen mögen. Fünfftens. Wellicher ein gantze grechtigkeit besitzt und kein vych zu erhalten vermag, der soll mögen syn rechtsamme zu einer ku uff die allment und zu einem rind uff die esch verliehen oder durch entlehnendes vych gebruchen." - War die wirtschaftliche Stellung der Halbbauern besser als diejenige der Tauner, so schloß dies doch nicht aus, daß beide sich gemeinsam für ihre Interessen wehrten. So erging unterm 13. April 1597 ein Spruch in einem Streit ,zwüschendt den tagneren und halben puren zu Buchs eins- und den übrigen puren, so die sechs höff daselbs zu Buchs buwend, anderstheils" über die Holznutzung in der Gemeinde Buchs. ${ }^{1}$ ) Es ist hier auch auf einen Streit über die Allmende in Altstetten zwischen den Bauern, ,so mit dem zug buwend" einerseits und den ,,minderen puren, so ire güter um den pfening und sonst bewerbend", sowie den Taunern andererseits hinzuweisen. ${ }^{2}$ ) Derselbe wurde durch Spruch vom 30. Juli 1578 beigelegt. Am 17. November 1797 erhielt die Gemeinde Altstetten ein bemerkenswertes Regulativ betreffend das Gemeindewerk (die den Gemeindegenossen obliegenden Dienstleistungen im Interesse der Gemeinden). ${ }^{3}$ ) Auch da ist der Bauer, weil er die größeren Gemeindedienste leistet, nämlich die Gemeindefuhren verrichtet, bei der Allmendnutzung bevorzugt. $\mathrm{Zu}$ den Gemeindefuhren wurden keine Kühe zugelassen, sie mußten vielmehr mit 3 Stieren verrichtet werden. Das Regulativ gab daher den Halbbauern, von denen offenbar nicht alle über Stiere verfügten, den Rat, zur Bestellung der Gemeindefuhren zusammenzustehen. Die Tatsache, daß die Dienstleistung des Tauners für die Gemeinde weniger wichtig war als diejenige des Bauern, war nun aber in Altstetten nicht nur bei der Verteilung des Allmendnutzens von Einfluß - die Bauern hatten zum voraus Anspruch auf den Nutzen eines gewissen Waldes --, die Buße des sich nicht zum Gemeindewerk einfindenden Tauners war dafür auch bedeutend milder als

1) Rechtsquellen des Kantons Zürich, 1. Teil, II S. $199 \mathrm{ff}$.

2) Ebenda I S. $304 . \quad$ 3) Ebenda I S. 312/13. 
diejenige, welche der ausbleibende Bauer auf sich lud. Sie betrug für diesen 1 t., für jenen $12 \beta$.

III. Wir haben bisher gesehen, wie bei Bestimmung des Umfanges der Allmendnutzung auf den Besitz von Zugvieh gesehen wurde. Der Mangel an Zugvieh ist aber nach den Quellen nicht der einzige Grund, warum eine gewisse Volksklasse, eben die Tauner, in der Nutzung hintangesetzt wurde. - Es ist zunächst darauf zu verweisen, daß nicht iiberall die Allmendnutzung prinzipiell sämtlichen Gemeindegenossen zustand. Der Anteil an der Allmend hatte vielmehr mancherorts von Anfang an den Besitz gewisser Güter zur Voraussetzung. Es sind dies Verhältnisse, welche sich aus der grundherrlichen Hofverfassung heraus entwickelt haben. $\left.{ }^{1}\right)$ Wer nicht ein solches bevorrechtetes Gut bebaute, besaß zunächst keinen Anspruch auf die Nutzung des diesen Gütern dienenden Allmendlandes. Diese Bindung der Nutzungsrechte an bestimmte Privatgüter kommt, wie v. $W y \beta$ ausführt, besonders häufig im bernischen Mittelland vor, in andern Kantonen, wie zum Beispiel in Zürich, nur vereinzelt. In Zürich nähern sich dieser Form nach v. Wy $\beta$ u. a. die Gemeinden Albisrieden und Schwamendingen. ${ }^{2}$ ) Wir haben denn auch besonders in den Rechtsquellen von Albisrieden in diesen Zusammenhang gehörende Bestimmungen gefunden. Albisrieden bildete ursprünglich eine geschlossene Grundherrschaft' höriger Leute des Großmünsters in Zürich. Die Chorherren verzichteten jedoch 1526 auf die hohen und niedern Gerichte in Albisrieden, hingegen verblieben ihnen ihre Zinsen und Lehen. Die Besitzer der ,Zinshöfe“" und „Huben" wollten nun gegenüber ihren Gemeindegenossen, ,so die tagner gnempt wurden", hinsichtlich des Weidganges auf bestimmten Wiesen ein Vorrecht haben, ,diewyl der mertheil vermelter wisen iren höfen zugehörig, ouch sy dieselben gedachtem gestifft (Großmünster) schwarlich verzinsen" mußten. Dieser Streit wurde durch einen Vergleich vom 15. September 1562 beigelegt. ${ }^{3}$ ) Auch

1) Vgl. hierüher v. Wy B, a. a. O. S. $103 \mathrm{ff}$.

2) A. a. O. S 104 Anm J.

3) Rechtsquellen des Kantons Zürich, 1. Teil, I S. 143 ff. 
bei dieser Abschließung einer Klasse von Vollberechtigten waren im Resultat die Tauner von der Nutzung nicht gänzlich ausgeschlossen. Es kam zur Ausbildung besonderer ,Taunerrechte". ${ }^{1}$ ) -- Auch in Schwamendingen hatten offenbar neben den Hubern die Tauner ebenfalls Anteil am Holznutzen: Nach der Offnung hatte derjenige, welcher aus dem Dorfe Holz hinwegführte und verkaufte, Strafe zu gewärtigen (eine beim Holznutzen häufig vorkommende Bestimmung); dabei hieß es, ,und soll $\mathrm{da} ß$ den huebren und Tagnauwren gleich gehalten werden". ${ }^{2}$ ) - In der Gemeinde Buchs gab es Waldungen, deren Nutzung sechs bestimmten Höfen zustand. Von diesen Waldungen mußten die Bauern, welche die sechs Höfe innehatten, den Vogthaber geben und machten nun in einem Streite mit den Taunern wegen der Holznutzung geltend, aus jenen Waldungen dürfe nur derjenige Holz beziehen, welcher den Vogthaber gebe, folglich hätten die Tauner auch kein Recht auf Holz. Die Tauner ließen sich dies denn auch ohne weiteres gefallen. ${ }^{3}$ ) Daneben gab es nun aber in Buchs noch Gemeindewaldungen. Über die Nutzung an diesen erging am 15. April 1562 ein Spruch, wonach das jährlich zu beziehende Holz in sieben gleiche Teile zerlegt wurde, wovon dann sechs Teile auf die sechs Höfe fielen und der siebente Teil den Taunerr zukam. Daneben wurde den Taumern das schon von jeher gehabte Recht, die ,affterschleg und windfäl“"4) und die Eicheln in den gemeinen Waldungen aufzulesen, von neuem zuerkannt.5) Ein weiterer Spruch betreffend die Holznutzung der Bauern, d. h. der Inhaber der sechs von vornherein privilegierten Höfe, und der Tauner erging am 13. April 1597.6) - Auch in Dielsdorf war denjenigen, welche Güter besaßen und den Vogthaber gaben, ein gewisser Wald zur alleinigen Nutzung vorbehalten, während weitere Waldparzellen der ganzen Gemeinde, Baucrn und Taunern zugleich dienten. ${ }^{7}$ )

1) Vgl. v. WyB a. a. O. S. 105 Anm. 2.

2) Schaubergs Zeitschr. f. noch ungedr. Rechtsquellen I S. 131.

$\left.{ }^{3}\right)$ Rechtsquellen des Kantons Zürich, 1. Teil, II S. 195/196.

4) Dürres Abfallholz.

5) Rechtsquellen des Kantons Zürich, 1. Teil, II S. 196/197.

$\left.{ }^{6}\right)$ Ebenda II S. $199 \mathrm{ff}$. 7) Ebenda II S. 339. 
IV. Es wurde im Vorhergehenden die Verschiedenartigkeit in der Nutzung am Allmendland auf herrschaftliche Verhältnisse zurïckgeführt. Im Kanton Zürich ist nun allerdings die Abschließung der Nutzungsbefugnisse zumeist aus anderen Ursachen hervorgegangen, wobei es aber zuletzt ebenfalls zur Ausbildung bestimmter Gerechtigkeiten kam. In Anlehnung an den in den Dorfoffnungen aufgestellten Grundsatz, daß Wohnsitz und eigener Rauch innert des Dorfetters genügte, um an den Nutzungen des Allmendlandes teilzunehmen, bildeten sich als Pertinenzen von Wohnhäusern dingliche Gerechtigkeiten aus. Die Nutzungsrechte verbanden sich hier nicht mit den Gütern, sondern mit den Häusern. ${ }^{1}$ ) An sich war da also auch der kleine Bauer, der Tauner, der ein eigenes Haus besaß, von der Nutzung nicht ausgeschlossen. Doch bildeten sich da ebenfalls Unterschiede im Umfang der Nutzungen heraus, der alte Gegensatz zwischen Bauern und Taunern kam auch hier zur Geltung.

Es ist davon auszugehen, daß nur da der Umfang der Nutzung im Belieben der einzelnen stehen konnte, wo die Größe und der Ertrag des Gemeinlandes allen Anforderungen gewachsen war. Dies kam aber mit der fortschreitenden Zeit, die eine Vermehrung der Bevölkerung und ein stetiges Anwachsen des Bedarfes brachte, immer weniger vor. ${ }^{2}$ ) Die bloße Beschränkung der Nutzung auf diejenigen, welche nutzungsberechtigte Hofstätten innehatten, genügte da nicht mehr, die einzelnen Berechtigungen mußten abgestuft werden. In einem Streit über den Weidgang in Dorlikon (Thalheim a.d. Thur) bemerkten die Bauern: „Die tagnoüwer clagtend sich unbillicher wyse ab inen, dann manicher tagnöwer vech uf die allmenten schlüge und daruff erhielte, der aber nüt einer handbreit weder in holtz noch in veld hette, dargegen sy vil wysen und ächer umb ein thür und

1) Vgl. hierüber v. Wy B a. a. O. S. $110 \mathrm{ff}$.

2) Nicht für jede Gemeinde galt, was 1675 Landvogt Steiner vom Gemeindegut in Dielsdorf berichtete: ,1. Daß ein jeder inwohner ... sovil veech, als er vermögenlich, uff die alment gmeinen weidgangs zu scblachen befügt, welliche von gwaltiger wy te und erthragenlicher nutzbarkeit" (Rechtsquellen des Kantons Zürich, 1. Teil, II S. 345). 
groß gelt erkoufft und davon schwer zins geben müßten, welche sy - wo man inen ir vech abgkante - nüt nach notturfft buwen und bewerben möchten." ${ }^{1}$ ) Solche Klagen gegen die Tauner stehen nicht vereinzelt da. Es lag auf der Hand, daß man, um allen Bedürfnissen zu genügen, dazu kam, bei Festsetzung des Umfanges der Nutzung auf die Größe des Sonderbesitzes zu schauen. So wurde denn in Dorlikon wie auch anderswo erklärt, daß ,einer wol vech, sovil er mit synem eignen höw und strow winteren möge, uf die allmenten schlachen und daruf erhalten dörffe ... "2) Es durfte also niemand mehr Vieh sömmern, als er auf seinem Gute wintern konnte. Die Tauner, welche keine eigene Landwirtschaft betrieben, waren somit bei einer strikten Durchführung dieses Grundsatzes vom Weidgang völlig ausgeschlossen. Dies wird denn auch in einem Vergleich zwischen den Bauern und Taunern in Benken vom Jahre 1604 hinsichtlich der ,tagnouwer, so weder wissen nach ächer habend", ausdrücklich gesagt. ${ }^{3}$ ) Die Abstufung der Nutzung nach dem Viehbestand nähert sich im Resultat derjenigen nach der Anzahl von Zugvieh, ohne sich jedoch ganz mit ihr zu decken, denn nur ganz bestimmte Haustiere kamien als Zugvieh in Frage. - Auch bei der Zuteilung des Holzes gelangte man dazu, auf den Bedarf zu schauen. In Dielsdorf wurde in der Ordnung betreffend die Holznutzung vom Jahre 1571 zwar anerkannt, daß der „Fronwald“ und derjenige ,,am Berg“"Gemeindewald sei, „also das rych und arm daryne theil habent". Trotzdem wurde dem Bauern, ,so ein gwerb (hat) und deßhalb vil volcks haben muß", je ein ganzer Hau ausgegeben, während je zwei Taunerhäuser sich in einen solchen teilen mußten. ${ }^{4}$ ) - Wurden den Taunern hinsichtlich des Maßes der Nutzung Schranken gezogen, so konnten sie sich doch im allgemeinen nicht über eine unbillige Behandlung beklagen. In einem Streite zwischen den Bauern und Taunern in Affoltern a. A. betreffend die Holznutzung im Homberg verglichen sich die Parteien unter Mithilfe der Obrigkeit, daß den Taunern

1) Rechtsquellen des Kantons Zürich, I Teil, II S. 432.

2) Ebenda S. 433 (1). $\quad$ 3) Ebenda I S. 468.

4) Ebenda II S. 340 (3). 
ein Teil des Homberg, dessen Waldbestand kahlgeschlagen worden war, überlassen wurde. Dies wurde den Taunern ,in ansechen der schweren thüri, damit sy sich mit iren wyb und kinden dester baß erneren mögen und das allmusen und biderb lüt destminder über louffen müßten, $u ß$ gnaden verwilligt und zugelassen". ${ }^{1}$ ) Hotz in seiner Untersuchung über die Rechtsverhältnisse an der Allmende Horger-Egg stellt geradezu den Satz auf, daß den Taunern im Gegensatz zu den Bauern durchgängig eine beträchtlichere Weidnutzung zukommt, als es bei scharfer Auslegung der zumeist gegen die ,richen“" gerichteten Maximalschranke der Nutzung der Fall wäre. ${ }^{2}$ ) In der einzigen uns bekannten, für das ganze Kantonsyebiet bestimmten Festsetzung der Nutzungsbefugnisse der Tauner und der ihnen gleichgestellten Handwerker und Gewerbetreibenden, in der Allmendverordnung für Zürich aus der Zeit des Bürgermeisters Hans Waldmann ${ }^{3}$ ), konnten die Tauner, die nicht einen halben Zug besaßen, ferner die Schmiede und die Müller folgende Tiere auf die Allmende treiben: zwei Kühe und ein Kalb oder zwei Kühe und ein Pferd, nebst zwei Schweinen und fünf Hühnern mit dem dazu gehörenden Hahn, hingegen keine Gänse. Für einen Kleinbauern, der nicht einmal einen halben Zug Vieh hatte, muß ein solcher Auftrieb als durchaus genügend bezeichnet werden. Auch das Regensberger Herrschaftsrecht von 1538, das im Gegensatz zu andern Amtsrechten eine Bestimmung über die Tauner enthält, war diesen hinsichtlich des Weidganges günstig gesinnt. Art. 117 lautet: „Item eyn Tagner mag han eyn Roß, eyn kug, und eyn kalb, darzu vier Süw und zechen hüner, Mit sölchen fugen so eyn tagner eygen gewächs hette wievil er dann vech von synem eygnen gut gewinteren, sovil mag er ouch Sünneren, doch soll er keyn futer kouffen, Aber die Armen Tagner, die gar keyn eygen gewechs haben, Mögennd wol futer kouffen, doch nit wytter, dann zu eym Roß, zu eyner kug und zu eynem kalb.“"4) Es wird hier

1) Rechtsquellen des Kantons Zürich, I S. 76 (1). 2) S. 16.

$\left.{ }^{3}\right)$ Es ist dies das eingangs erwähnte Erkenntnis von Bürgermeister und Rat.

4) Pestalutz, Vollständige Sammlung der Statute des Kantons Zürich, I S. 214. Vgl. auch den erwähnten Spruch, betr. die Holzgerechtig- 
also zugunsten der kein eigenes Wiesland für die Winterung von Vieh besitzenden Tauner der Grundsatz, daß der Umfang des Allmendauftriebes sich nach der Tragfähigkeit des Sonderbesitzes richte, verlassen.

Haben wir in der erwähnten Allmendverordnung, die allerdings vermutlich nur Entwurf geblieben ist ${ }^{1}$ ), und in der aufgeführten Bestimmung aus dem Regensberger Herrschaftsrecht wenigstens Ansätze zu einer allgemein gültigen Regelung des Weidganges der Tauner, so fehlen hinsichtlich der Holznutzung solche über das Gebiet einer einzelnen Gemeinde hinausgehende Frlasse. Dies wohl nicht zum wenigsten darum, weil die Abschließung der Holznutzung lange Zeit nicht in dem Maße dringend war wie diejenige des Weidganges. Hinsichtlich der Regelung des Umfanges der Holznutzungen innerhalb der einzelnen Gemeinden ist auf die bereits gegebenen Beispiele zu verweisen. Wir haben bei Dielsdorf gesehen, daß die Zuteilung dès Holzes an die Bauern doppelt so groß war wie diejenige an die Tauner. Dasselbe war nun auch in Dübendorf der Fall. Hier traf es anf die ,Bauernhausgerechtigkeit" zwei und auf die ,,Taunergerechtigkeit" ein Klafter Holz aus den Gemeindewaldungen. ${ }^{2}$ ) Im Gegensatz zum Weidgang scheint cine weitergehende Abstufung bei den Holzbezügen nicht vorgekommen zu sein.

V. Wie überhaupt hinsichtlich der Allmendnutzung, so wurde auch bei den Taunern, die Anspruch auf Nutzung machten, darauf gesehen, daß sie auch gewisse persönliche Erfordernisse erfüllten: daß sie Gemeindegenossen waren. So hatten zum Beispiel in Dielsdorf die Tauner, die lediglich Hintersassen waren, keinerlei Anspruch auf Zuteilung von $\mathrm{Holz}^{3}$ ) Waren die Tauner nun aber in allen Gemeindeangelegenheiten vollberechtigte Gemeindegenossen oder kam

keit der Tauner in Buchs von 1562, Rechtsquellen des Kantons Zürich, I. Teil, II S. 198 (I.0).

1) Vgl. v. Wy B a. a. O. S. 93 Anm. I.

2) Rechtsquellen des Kantons Zürich, 1. Teil, II S. 482. Ehedem bezog in Dübendorf der Bauer 7, der Tauner 3 Klafter, später wegen Holzmangel nur noch 5 bzw. 2, bis man auf das Verhältnis von 2 zu 1 kam. Siehe ebenda II S. 481 Anm. 1.

3) Ebenda II S. 340 . 
ihre besondere wirtschaftliche Stellung auch bei Festsetzung ibrer politischen Rechte innerhalb der Gemeinde zum Ausdruck? Es ist davon auszugehen, daß die Zahl der Tauner diejenige der Bauern überstieg oder doch stetig anwuchs. So gab es zum Beispiel in Dübendorf im Jahre 1686 ca. 18 Bauern und ca. 27 Tauner. ${ }^{1}$ ) Die Tauner waren daher nicht nur bei der Ausübung der Allmendnutzung selbst den Bauern überlegen oder zum mindesten ebenbürtig, wenn man nur auf die Kopfzahl Bedacht nahm, sondern auch bei der Ordnung der auf die Allmende bezüglichen Verhältnisse. Auch hier fand man es somit für nötig, die Bauern gegenüber den Taunern zu bevorzugen. Ganz ausgeschlossen waren die Tauner bei der Bestellung der in der Allmendverordnung aus der Zeit Hans Waldmanns vorgesehenen drei oder fünf Geschworenen zur Beaufsichtigung der Allmend. Obwohl die Tauner in jener Allmendverordnung, wie wir sahen, sachlich durchaus keine schwere Zurücksetzung crfuhren, so hatten sie doch hier weder das aktive Wahlrecht, noch konnten sie gewählt werden. ${ }^{2}$ ) Doch war die Regierung den Taunern hinsichtlich ihrer Stellung als wahlberechtigte und wahlfähige Gemejndeglieder richt stetsfort so ungünstig gesinnt. In der Holzordnung vom Jahre 1549, welche die Regierung der Gemeinde Opfikon gab, hatte die Gemeinde von den drei Verordneten uiber die Allmende wenigstens einen aus der Zahl der Tauner zu wählen. ${ }^{3}$ ) In einem Streit zwischen den Bauern und Taunern in Dübendorf bestimmten die Vertreter der Regierung im Jahre 1686, daß der Abnahme der Gemeinderechnung künftig zwei Vertreter der Tauner, ,ohne der gmeind kosten" beiwohnen durften. ${ }^{4}$ ) In dem schon mehrfach erwähnten Vergleich zwischen den Bauern und Taunern von Affoltern am Albis über die Holznutzung im Homberg vom Jahre 1563 wurde bestimmt, daß an der Gemeindeversammlung diejenigen, welche weder Haus noch Heim hatten, nicht teilnehmen konnten. Veranlassung zu dieser Bestim-

1) Rechtsquellen des Kantons Zürich, 1. 'Teil, II S. 481 Anm. 1.

2) Schaubergs Zeitschr. f. noch ungedr. Rechtsquellen I S. 136.

3) Ebenda I S. 134.

4) Rechtsquellen des Kantons Zürich, 1. Teil, II S. 481. 
mung gab folgende Klage der Bauern: „... w was jedem von inen (den Bauern) guter gethrüwer meinung, der gmeind zu nutz und gutem angesechen werde, und es den tagnern nit gfalle, haltind sy (die Tauner) ein andere gmeind und ubermerind sy, dann iren bi drig und dryßigen, die weder huß noch heim ald einer handtbreit habind; die ordnind und machind dann in söllichen der gmeind sachen, es sige ze rüthen als in ander weg, nach irem gfallen...."1) Auch in Benken scheinen die Tauner ihre Mehrheit bei den Gemeindeversammlungen entgegen dem gemeinen Interesse ausgenützt zu haben. In dem Verglejch vom Jahre 1619 wurde ihnen nun zwar das Stimm- und Wahlrecht an der Gemeindeversammlung nicht geschmälert, doch wurden sie aufgefordert, ,,das sy darinn ein bescheidenheit halten und nit ein mehr machen söllind, dadurch man umb holtz und boden kemme".${ }^{2}$ )

Die Betrachtung über die Stellung der Tauner in Allmend und Gemeinde schließend, sei nun noch folgende bemerkenswerte Bestimmung aus der Ordnung betreffend die Bauern und Tauner in Dielsdorf vom Jahre 1571 aufgeführt: ,So und wenn aber einer, der in der gmeind Dielstorff wonhafft ist, gegen einem anderen ingsessnen gmeindtsgnossen daselbs syn huß und heim verkouffte, alsdann derselkig verköuffer die ernüwerung der dorffsgrechtigkeit nit schuldig syn, sonders in der gmeind als ein gmeindtsgnoß wol fürer blyben mögen, doch in den nutzungen der gmeind wie ein tagnower gehalten werden.“ ${ }^{3}$ )

Wir haben in obigem die Bauern und Tauner als in einem sachlich mehr oder weniger schroffen Gegensatz zueinander stehende Bevölkerungsschichten in den zürcherischen Landgemeinden kennengelernt. Genossen derselben Gemeinde, schlossen sie sich, wie die Quellen zeigen, doch voneinander ab, so daß man das Gefühl hat, als zerfalle die eine Gemeinde wieder in zwei nicht dem Raume, wohl aber den Personen nach getrennte Unterabteilungen. Von seiten der Regierung wurden die beiden Gruppen der Bauern und Tauner, wie die mannigfachen

1) Rechtsquellen des Kantons Zürich, 1. Teil, I S. 80 (8).

2) Ebenda I S. $469 . \quad$ 3) Ebenda II S. 342 (11). 
Die Stellung d. Tauner n. d. Rechtsquellen d. Kantons ZZ̈̈rich. 129

Rechtssprüche und Vergleiche erweisen ${ }^{\mathbf{1}}$ ), ohne weiteres als Parteien in den häufigen Streitigkeiten über die Allmendnutzungen anerkannt. ${ }^{2}$ ) Die Obrigkeit ließ es jeweilen nicht an Ermahnungen fehlen, sie sollten, gegen einandern gut fründ, nachpuren und gmeindsgnossen syn und einandern deß zu argem ald ungutem niemer mer gedencken in dhein $\left.\mathrm{wy}^{3}{ }^{\mathrm{s}}{ }^{3}\right)$

1) Der Einblick in diese Verhältnisse ist durch die Publikation der Rechtsquellen des Kantons Zürrich, welche in ihrem ersten Teile die Rechtsquellen einer Landgemeinde nach der anderen in alphabetischer Reihenfolge bringt, ungemein erleichtert worden, obwohl der zweite Band erst bis zur Gemeinde Dürnten reicht.

2) In dem Spruch, betr. die Holzgerechtigkeit der Tauner in Buchs aus dem Jahre 1554 werden die Tauner insgesamt z. B. als Kläger, die Bauern als Antworter bezeichnet (Rechtsquellen des Kantons Zürich, 1. Teil, II S. 193). - Über die Tragung der Kosten des Verfahrens vgl. z. B. Rechtsquellen des Kantons Zürich, 1. Teil, I S. 146 (5). - Über eine von Taunern zu Auslikon gegen einen Entscheid des Gerichtes zu Greiffensee ergriffene Appellation siehe Rechtsquellen des Kantons Zürich, 1. Teil, I S. 326 Anm.

3) Rechtsquellen des Kantons Zürich, 1. Teil, I S. 146 (5). Vgl. auch ebenda II S. 407 (25). 